\title{
Research Paper: Association of SHANK3 Gene Polymorphism and Parkinson Disease in the North of Iran
}

\author{
Nahid Mizban ${ }^{1}$ (D), Nasim Vousooghi ${ }^{*}$ (D), Nasrin Mizban $^{3}$ (D) \\ 1. Department of Biology, Faculty of Sciences, University of Guilan, Guilan, Iran. \\ 2. Department of Applied Cell Sciences, School of Advanced Technologies in Medicine, Tehran University of Medical Sciences, Tehran, Iran. \\ 3. Department of Biological Sciences, Faculty of Biological Sciences and Technologies, Shahid Beheshti University, Tehran, Iran.
}

\begin{tabular}{|l|l|l}
\hline $\begin{array}{c}\text { Use your device to scan } \\
\text { and read the article online }\end{array}$ \\
the North of Iran. Basic and Clinical Neuroscience, 12(1), 57-62. http://dx.doi.org/10.32598/bcn.12.1.255.4 \\
dof http://dx.doi.org/10.32598/bcn.12.1.255.4
\end{tabular}

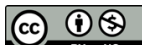

Article info:

Received: 6 Jan 2018

First Revision: 25 Feb 2018

Accepted: 30 Apr 2018

Available Online: 01 Jan 2021

Keywords:

SHANK3, Parkinson disease, Gene polymorphism, rs9616915

\section{A B S T RA C T}

Introduction: Parkinson Disease (PD), the second most common chronic neurodegenerative disorder, is characterized by tremor, bradykinesia, rigidity, and postural instability. SHANK3 (SH3 and multiple ankyrin repeat domain 3) belongs to the extremely conserved ProSAP/ Shank family of synaptic scaffolding proteins. Meanwhile, rs9616915 is a non-synonymous SNP $(\mathrm{T}>\mathrm{C})$ located in the exon 6 of the SHANK3 gene, which induces substitution of isoleucine to threonine and affects the function of the resulted protein. The present study aimed to evaluate whether rs9616915 polymorphism of SHANK3 is involved in the susceptibility to PD.

Methods: The study subjects were 100 patients diagnosed with PD and 100 control volunteers. The obtained samples were evaluated by the polymerase chain reaction-restriction fragment length polymorphism method.

Results: A significant association was found in genotype distribution between cases and controls. Individuals with TC genotype had increased risk of $\mathrm{PD}(\mathrm{P}=0.035, \mathrm{OR}=1.98,95 \%$ $\mathrm{CI}=1.04$ - 3.74). No significant difference was found in allele distribution $(\mathrm{P}=0.7)$.

Conclusion: The findings suggest that the SHANK3 rs 9616915 polymorphism is associated with an increased risk of PD in the population. Further studies are needed to confirm the role of the SHANK3 gene in PD.

\section{* Corresponding Author:}

Nasim Vousooghi, Pharm D, PhD.

Address: Department of Applied Cell Sciences, School of Advanced Technologies in Medicine, Tehran University of Medical Sciences, Tehran, Iran. Tel: +98 (912) 3724378

E-mail:n-vousooghi@tums.ac.ir 


\section{Highlights}

- The SHANK3 rs9616915 polymorphism is associated with an increased risk of PD.

- Individuals with TC genotype were at higher risk of developing PD.

\section{Plain Language Summary}

Parkinson's Disease (PD) is the second most common chronic neurodegenerative disease of aging people that affects $1-2 \%$ of the population over 60 . It is well known for tremor, rigidity, and postural instability, often leading to falling and injury. People with PD are especially susceptible to hip fractures with a 3.2-fold greater risk than other people. It has been shown that some genes and their variations have a crucial role in disease recognizing. Previous studies have shown that a gene called SHANK3 and its variations play an important role in susceptibility to some neurodevelopmental disorders, which led us to hypothesize that functional polymorphisms within the SHANK3 gene might be linked to susceptibility to PD. To our knowledge, this is the first genetic variation study on the association between the SHANK3 gene, its polymorphic regions, and the risk of PD. Our results of a case-control study in the north of Iran showed that a SHANK3 gene variation called rs9616915 is associated with an increased risk of PD, and individuals with TC genotypes were 1.9-fold at a higher risk of developing the disease. Further studies are needed to confirm the role of the SHANK3 gene in PD.

\section{Introduction}

arkinson Disease (PD), the second most $\mathbf{P}$ common chronic neurodegenerative disorder in aging people, affects $1 \%-2 \%$ of the population over 60 . It is characterized by tremor, bradykinesia, rigidity, and postural instability, leading to falling and injury (Schapira, 1999). Loss of dopaminergic neurons and lower brain dopamine activity in PD patients has been reported in pathological investigations (Shannak et al., 1994). The progressive degeneration of dopaminergic neurons in the substantia nigra leads to motor impairment and the manifestation of disease symptoms (Frenklach, Louie, Koop, \& Bronte-Stewart, 2009). This may explain why dopaminergic therapy can improve the tremor and rigidity in PD patients (Rothermel \& Garcia, 1972). Although it has been shown that synaptic defects are involved in PD, the exact mechanism is still unknown.

SHANK3 (SH3 and multiple ankyrin repeat domain 3) is one of the most promising genes involved in neuronal function. SHANK3 protein is a vital scaffolding protein of the Postsynaptic Density (PSD) of excitatory glutamatergic synapses in the Central Nervous System (CNS) (Kreienkamp, 2008). The gene is located on chromosome 22q13.3, spans about $58 \mathrm{~kb}$, and comprises 24 exons, 7 of which are alternatively spliced (Durand et al., 2007). The product of SHANK3 gene expression is a large protein containing diverse domains such as an ANK repeat, a proline-rich cluster, $\mathrm{SH} 3, \mathrm{PDZ}$, and SAM (sterile a-motif) (Kreienkamp, 2008). This molecule directly or indirectly interacts with cytoskeletal proteins and neurotransmitter receptors. It is also involved in the formation, expansion, and development of dendritic spines and is an essential factor for developing functional synapses (Roussignol et al., 2005). Different mutations in the sequence of the SHANK3 gene may disrupt the structure and function of gene domains (Mameza et al., 2013), and the resulting variants may be associated with various brain disorders. Non-synonymous/frameshift and deletion/duplication mutations in SHANK3 have been detected in PD patients (Wilson et al., 2003) It has been reported that a non-synonymous polymorphism named rs9616915 ( $>C$ C) may occur in the exon 6 of the SHANK3 gene, which induces an amino acid replacement, i.e. isoleucine to threonine. This alteration directly affects the gene function of splicing regulation and results in protein structure damage (Shao et al., 2014). In this study, we aimed to evaluate the association between (rs9616915) SHANK3 polymorphism and susceptibility to Parkinson disease in people living in the north of Iran.

\section{Materials and Methods}

\subsection{Patients and controls}

A total of 100 patients with PD (76 males) and 100 healthy controls participated in the study. The controls and patients were unrelated subjects selected from the same population living in Guilan Province, north of Iran. The patients' characteristics were collected from 
Iran Medical Diagnostic Center in Rasht City, Iran. The subjects' age ranges between 60 and 80 years. The participants were investigated in terms of having certain genetic diseases in close relatives. Also, they would be excluded from the study if they had a history of any other neurological disorder, acquired brain injury, or severe mental illness. Each subject gave a 2-mL blood sample drawn into Ethylenediaminetetraacetic Acid (EDTA)coated tubes (Venoject, Belgium) for the next step of genomic DNA extraction. The local Ethics Committee has approved this study, and written consent was obtained from all participants.

\subsection{Genotyping}

Genomic DNA was extracted from whole-blood samples using DNA Extractor Gpp Solution Kit (Gen Pajoohan, Iran) according to the manufacturer's instructions. The intended SNP (rs9616915 in the SHANK3 gene) was evaluated by polymerase chain reaction-restriction fragment length polymorphism assay. Primer sequences for PCR reactions were 5'-GCTTGACACCCCTCTACCA -3' (forward) and 5' -TCTGCCCCATAGAACAGC-3' (reverse). PCR reactions were performed in a total volume of $25 \mu \mathrm{L}$ and consisted of an initial denaturation step at $95^{\circ} \mathrm{C}$ for $5 \mathrm{~min}$ followed by 30 cycles of denaturation at $95^{\circ} \mathrm{C}$ for $30 \mathrm{~s}$ and annealing at $55^{\circ} \mathrm{C}$ for $30 \mathrm{~s}$. PCR products were subsequently digested by restriction enzyme hphl. After endonuclease digestion, products were separated on $2 \%$ agarose gel electrophoresis and visualized by ethidium bromide staining.

\subsection{Statistical analysis}

Statistical analysis was performed by MedCalc (version12.1, Mariakerke, Belgium). Analysis of differences in allele and genotype frequencies between cases and controls was done using the $\chi^{2}$ test. To estimate the association between the SHANK3 rs9616915 variant and the risk of Parkinson disease, the odds ratios with $95 \%$ confidence intervals $(95 \% \mathrm{CI})$ were evaluated by logistic regression. A value of $\mathrm{P}<0.05$ was considered statistically significant.

\section{Results}

Two hundred subjects, including 100 patients with Parkinson disease and 100 healthy volunteers as the control group, were evaluated in the study. The Mean \pm SD age of the cases at the time of diagnosis was slightly more than the control group ( $72.5 \pm 2.6$ vs. $70.2 \pm 2.1$ years, respectively), but the difference was not statistically significant ( $\mathrm{P}>0.05)$. Genotyping of rs9616915 was done by PCR-
RFLP method (Figure 1). Genotype frequencies for CC, TC, and TT were $33 \%, 50 \%$, and $17 \%$ in controls, and $24 \%, 72 \%$, and $4 \%$ in PD patients, respectively. Statistical analysis showed a significant difference between the two groups $(\mathrm{P}=0.001)$. The results indicated that the subgroup with TC genotype had an increased risk of Parkinson disease $(\mathrm{P}=0.035, \mathrm{OR}=1.98,95 \% \mathrm{CI}=1.04$ - 3.74). All information about allele and genotype frequencies and associated ORs $(95 \% \mathrm{CI})$ for cases and controls are summarized in Table 1. Besides, as most of our cases were male subjects (76 out of 100), we re-analyzed the data again for male cases. After removing the female genotypes from our population, the statistical analysis revealed that the results are consistent with females. The individuals with TC genotype had an increased risk of Parkinson disease $(\mathrm{P}=0.029$, $\mathrm{OR}=2.17,95 \% \mathrm{CI}=1.08-4.37)($ Table 2$)$.

\section{Discussion}

In this case-control study, we evaluated the relationship between rs9616915 polymorphism of SHANK3 gene and Parkinson disease in 100 cases and 100 control subjects. Our results showed a significant association in genotype distribution between cases and controls. Individuals with TC genotype were at higher risk (1.9 fold) of developing PD than other genotypes.

SHANK3 is a member of the highly conserved ProSAP/ Shank family of master synaptic scaffolding proteins (Qualmann, Boeckers, Jeromin, Gundelfinger, \& Kes-

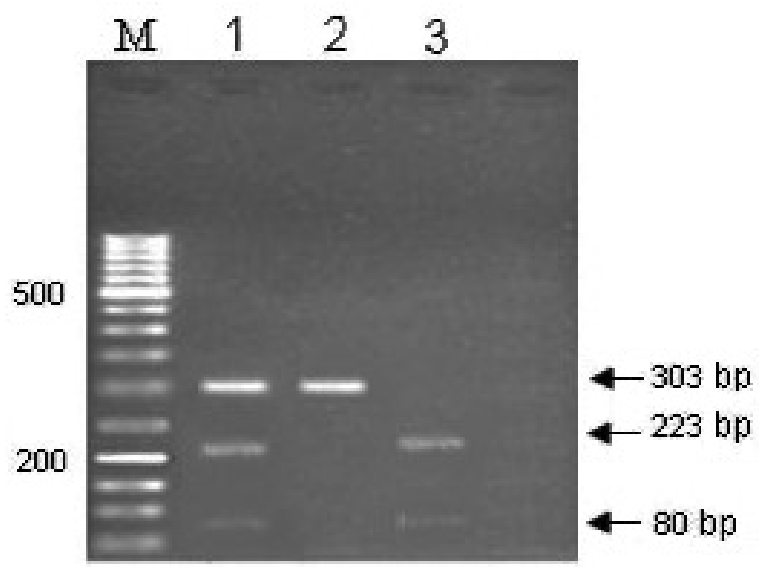

NEUR SCIENCE

Figure 1. Detection of SHANK3 gene polymorphism by polymerase chain reaction-restriction fragment length polymorphism (PCR-RFLP) using hph1 restriction enzyme

Lane 1, fragments showing genotype for heterozygous patients; lane 2, fragments indicating the TT genotype for the wild type homozygous patient; Lane 3, fragments presenting the CC genotype for the homozygous mutant patient; $\mathrm{M}=50 \mathrm{bp}$ DNA marker. 
Table 1. Allele and genotype frequencies of SHANK3 rs9616915 polymorphism among cases and controls and the associations with risk of Parkinson's disease $(n=100)$

\begin{tabular}{|c|c|c|c|c|c|c|}
\hline \multirow{2}{*}{\multicolumn{2}{|c|}{ Allele and Genotype Frequencies }} & \multicolumn{2}{|c|}{ NO. (\%) } & \multirow{2}{*}{ OR (95\% Cl) } & \multirow{2}{*}{$\mathbf{P}^{\mathbf{a}}$} & \multirow{2}{*}{$\mathbf{P}^{\mathbf{b}}$} \\
\hline & & Controls & Patients & & & \\
\hline \multirow{2}{*}{ Alleles } & C & $116(58)$ & $120(60)$ & 1.00 & 0.76 & - \\
\hline & $\mathrm{T}$ & $84(42)$ & $80(40)$ & $0.92(0.61-1.37)$ & - & 0.684 \\
\hline \multirow{4}{*}{ Genotypes } & CC & $33(33)$ & $24(24)$ & 1.00 & 0.001 & - \\
\hline & TC & $50(50)$ & $72(72)$ & $1.98(1.04-3.74)$ & - & 0.035 \\
\hline & $\mathrm{TT}$ & $17(17)$ & $4(4)$ & $0.32(0.09-1.08)$ & - & 0.067 \\
\hline & $\mathrm{TT}+\mathrm{TC}$ vs CC & $67(67)$ & $76(76)$ & $1.55(0.83-2.89)$ & - & 0.16 \\
\hline
\end{tabular}

${ }^{a}$ Allele and genotype frequencies in cases and controls were compared using $\chi^{2}$ test; ${ }^{b}$ Significance level for allele and genotype frequencies in cases and controls.

Table 2. Genotype frequencies of SHANK3 rs9616915 polymorphism among male cases and controls and the associations with risk of Parkinson's disease

\begin{tabular}{|c|c|c|c|c|c|}
\hline \multirow{2}{*}{ Genotypes } & Controls $(n=100)$ & Patients $(n=76)$ & \multirow{2}{*}{ OR $(95 \% \mathrm{Cl})$} & \multirow{2}{*}{$\mathbf{P}^{\mathbf{a}}$} & \multirow{2}{*}{$\mathbf{P}^{\mathbf{b}}$} \\
\hline & n (\%) & n (\%) & & & \\
\hline $\mathrm{CC}$ & $33(33)$ & $17(22)$ & 1.00 & 0.002 & - \\
\hline $\mathrm{TC}$ & $50(50)$ & $56(74)$ & $2.17(1.08-4.37)$ & - & 0.029 \\
\hline TT & $17(17)$ & $3(4)$ & $0.34(0.08-1.33)$ & - & 0.122 \\
\hline
\end{tabular}

${ }^{a}$ Allele and genotype frequencies in cases and controls were compared using $\chi^{2}$ test; ${ }^{b}$ Significance level for allele and genotype frequencies in cases and controls.

sels, 2004; Bozdagi et al., 2010). The reduced expression of $S H A N K 3$ results in the attenuation of the number of dendrites and synaptic transmission and plasticity. Malfunctions of synaptic transmission might result in brain disorders such as epilepsy, autism, Alzheimer, and Parkinson disease (Lepeta et al., 2016). It has been shown that the loss of SHANK3 in in vitro cultures of hippocampal neurons may decrease the number and augmented length of dendritic spines. In contrast, expression of SHANK3 could induce the formation of de novo functional dendritic spines in aspiny cerebellar neurons (Roussignol et al., 2005).

Moreover, the absence of one copy of the SHANK3 gene or its mutations could result in changes in spine morphology and behavioral issues related to autism traits such as deficits in social communication, social interaction, and repetitive actions (Bangash et al., 2011; Durand et al., 2012). Accomplished studies about this gene have indicated that genetic variations of SHANK3 play an essential role in susceptibility to many kinds of neurodevelopmental disorders. Meanwhile, rs9616915 polymorphism is a non-synonymous substitution from isoleucine to threonine in the exon 6 of $S H A N K 3$, which could interfere with the gene function via affecting the splicing process (Shao et al., 2014). Previous studies have reported its functional involvement in hippocampal mRNA expression of SHANK3 (Zhang, Wu, Hong, Peng, \& Fang, 2016). In a case-control study in China, it was reported that rs9616915 polymorphism is associated with decreased risk of Autism Spectrum Disorders (ASD) (Shao et al., 2014). However, other studies have not found an association between rs9616915 and ASD (Qin et al., 2009; Sykes et al., 2009; Jonsson et al., 2014). In another study, Mashayekhi, Mizban, Bidabadi, and Salehi (2016) reported that rs9616915 is accompanied by a higher risk of ASD.

The inconsistency may be related to different environmental conditions, relatively small sample size, varia- 
tions in diagnostic criteria of the disorder, and diverse ethnicity. For example, it has been stated that the $\mathrm{C}$ allele frequency is $48.1 \%$ in the European population while it is $3.5 \%$ among Han Chinese people (Zhang et al., 2016). In other brain disorders, it has been shown that the synaptic dysfunction and loss of glutamate receptors at the Shank-postsynaptic platform could contribute to Alzheimer disease (Gong, Lippa, Zhu, Lin, \& Rosso, 2009). A significant association between $S H A N K 3$ variation and Phelan-McDermid syndrome has also been reported (Bonaglia et al., 2011). Moreover, mutations in the SHANK3 gene are associated with schizophrenia (Gauthier et al., 2010). This mutation in bipolar disorder has also been investigated in previous studies (Zhang et al., 2016). These findings led us to hypothesize that a functional polymorphism within the SHANK3 gene might be linked to susceptibility to Parkinson disease. To our knowledge, this is the first study evaluating the association between $S H A N K 3$ gene polymorphism and the risk of Parkinson disease. However, it should be mentioned that this study has some limitations. We have only evaluated one SNP in the SHANK3 gene, which is not adequate to assess the risk of Parkinson disease in a subject, and many other genes and SNPs may also be involved in the risk of disease. Besides, our sample size was not so large, and further studies with larger sample sizes are needed in the future to confirm the role of the SHANK3 gene in Parkinson disease.

\section{Conclusion}

In conclusion, our results indicated that the SHANK3 rs9616915 polymorphism is associated with an increased risk of Parkinson disease. Further studies with more patients and controls are required to confirm our results.

\section{Ethical Considerations}

\section{Compliance with ethical guidelines}

The local Ethics Committee has approved this study, and written consent was obtained from all participants.

\section{Funding}

This study was supported by the University of Guilan.

\section{Authors' contributions}

Conceptualization and supervision: Nasim Vousooghi; Methodology: Nasim Vousooghi; Investigation, writing and validation: Nahid Mizban; Review \& editing: All authors; Formal analysis, Data and draft preparation: Nasrin Mizban

\section{Conflict of interest}

The authors declared no conflict of interest.

\section{Reference}

Bangash, M. A., Park, J. M., Melnikova, T., Wang, D., Jeon, S. K., \& Lee, D., et al. (2011). RETRACTED: Enhanced polyubiquitination of shank3 and NMDA receptor in a mouse model of Autism. Cell, 145(5), 758-72. [DOI:10.1016/j.cell.2011.03.052] [PMID] [PMCID]

Bonaglia, M. C., Giorda, R., Beri, S., De Agostini, C., Novara, F., \& Fichera, M., et al. (2011). Molecular mechanisms generating and stabilizing terminal 22q13 deletions in 44 subjects with Phelan/McDermid syndrome. PLoS Genet, 7(7), e1002173. [DOI:10.1371/journal.pgen.1002173] [PMID] [PMCID]

Bozdagi, O., Sakurai, T., Papapetrou, D., Wang, X., Dickstein, D. L., \& Takahashi, N., et al. (2010). Haploinsufficiency of the autism-associated Shank3 gene leads to deficits in synaptic function, social interaction, and social communication. Molecular Autism, 1(1), 1-15. [DOI:10.1186/2040-2392-1-15] [PMID] [PMCID]

Durand, C. M., Betancur, C., Boeckers, T. M., Bockmann, J., Chaste, P., \& Fauchereau, F., et al. (2007). Mutations in the gene encoding the synaptic scaffolding protein SHANK3 are associated with autism spectrum disorders. Nature Genetics, 39(1), 25-7. [DOI:10.1038/ng1933] [PMID] [PMCID]

Durand, C. M., Perroy, J., Loll, F., Perrais, D., Fagni, L., \& Bourgeron, T., et al. (2012). SHANK3 mutations identified in autism lead to modification of dendritic spine morphology via an actin-dependent mechanism. Molecular Psychiatry, 17(1), 71-84. [DOI:10.1038/mp.2011.57] [PMID] [PMCID]

Frenklach, A., Louie, S., Koop, M. M., \& Bronte-Stewart, H. (2009). Excessive postural sway and the risk of falls at different stages of Parkinson's disease. Movement Disorders, 24(3), 377-85. [DOI:10.1002/mds.22358] [PMID]

Gauthier, J., Champagne, N., Lafrenière, R. G., Xiong, L., Spiegelman, D., \& Brustein, E., et al. (2010). De novo mutations in the gene encoding the synaptic scaffolding protein SHANK3 in patients ascertained for schizophrenia. Proceedings of the National Academy of Sciences, 107(17), 7863-8. [DOI:10.1073/ pnas.0906232107] [PMID] [PMCID]

Gong, Y., Lippa, C. F., Zhu, J., Lin, Q., \& Rosso, A. L. (2009). Disruption of glutamate receptors at Shank-postsynaptic platform in Alzheimer's disease. Brain Research, 1292, 191-8. [DOI:10.1016/j.brainres.2009.07.056] [PMID] [PMCID]

Jonsson, L., Zettergren, A., Pettersson, E., Hovey, D., Anckarsäter, H., \& Westberg, L., et al. (2014). Association study between autistic-like traits and polymorphisms in the autism candidate regions RELN, CNTNAP2, SHANK3, and CDH9/10. Molecular Autism, 5(1), 1-9. [DOI:10.1186/20402392-5-55] [PMID] [PMCID] 
Kreienkamp, H. J. (2008). Scaffolding proteins at the postsynaptic density: Shank as the architectural framework. Protein-Protein Interactions as New Drug Targets, 365-80. [DOI:10.1007/978-3540-72843-6_15] [PMID]

Lepeta, K., Lourenco, M. V., Schweitzer, B. C., Martino Adami, P. V., Banerjee, P., \& Catuara-Solarz, S., et al. (2016). Synaptopathies: synaptic dysfunction in neurological disorders-A review from students to students. Journal of neurochemistry, 138(6), 785-805. [DOI:10.1111/jnc.13713] [PMID] [PMCID]

Mameza, M. G., Dvoretskova, E., Bamann, M., Hönck, H. H., Güler, T., \& Boeckers, T. M., et al. (2013). SHANK3 gene mutations associated with autism facilitate ligand binding to the Shank3 ankyrin repeat region. Journal of Biological Chemistry, 288(37), 26697-708. [DOI:10.1074/jbc.M112.424747] [PMID] [PMCID]

Mashayekhi, F., Mizban, N., Bidabadi, E., \& Salehi, Z. (2016) The association of SHANK3 gene polymorphism and autism. Minerva Pediatrica. [Online Ahead of Print] [PMID]

Qin, J., Jia, M., Wang, L., Lu, T., Ruan, Y., \& Liu, J., et al. (2009). Association study of SHANK3 gene polymorphisms with autism in Chinese Han population. BMC Medical Genetics, 10(1), 1-6. [DOI:10.1186/1471-2350-10-61] [PMID] [PMCID]

Qualmann, B., Boeckers, T. M., Jeromin, M., Gundelfinger, E. D., \& Kessels, M. M. (2004). Linkage of the actin cytoskeleton to the postsynaptic density via direct interactions of Abp1 with the ProSAP/Shank family. Journal of Neuroscience, 24(10), 2481-95. [DOI:10.1523/JNEUROSCI.5479-03.2004] [PMID] [PMCID]

Rothermel, J. E., \& Garcia, A. (1972). Treatment of hip fractures in patients with Parkinson's syndrome on levodopa therapy. Journal of Bone and Joint Surgery, 54(6), 1251-4. [DOI:10.2106/00004623-197254060-00013]

Roussignol, G., Ango, F., Romorini, S., Tu, J. C., Sala, C., \& Worley, P. F., et al. (2005). Shank expression is sufficient to induce functional dendritic spine synapses in aspiny neurons. Journal of Neuroscience, 25(14), 3560-70. [DOI:10.1523/JNEUROSCI.4354-04.2005] [PMID] [PMCID]

Schapira, A. H. (1999) Science, medicine, and the future: Parkinson's disease. The BMJ, 318, 311-4. [DOI:10.1136/ bmj.318.7179.311] [PMID] [PMCID]

Shannak, K., Rajput, A., Rozdilsky, B., Kish, S., Gilbert, J., \& Hornykiewicz, O. (1994). Noradrenaline, dopamine and serotonin levels and metabolism in the human hypothalamus: observations in Parkinson's disease and normal subjects. Brain Research, 639(1), 33-41. [DOI:10.1016/0006-8993(94)91761-2]

Shao, S., Xu, S., Yang, J., Zhang, T., He, Z., \& Sun, Z., et al. (2014). A commonly carried genetic variant, rs9616915, in SHANK3 gene is associated with a reduced risk of autism spectrum disorder: Replication in a Chinese population. Molecular Biology Reports, 41(3), 1591-5. [DOI:10.1007/s11033-013-3005-5] [PMID]

Sykes, N. H., Toma, C., Wilson, N., Volpi, E. V., Sousa, I., \& Pagnamenta, A. T., et al. (2009). Copy number variation and association analysis of SHANK3 as a candidate gene for autism in the IMGSAC collection. European Journal of Human Genetics, 17(10), 1347-53. [DOI:10.1038/ejhg.2009.47] [PMID] [PMCID]

Wilson, H. L., Wong, A. C. C., Shaw, S. R., Tse, W. Y., Stapleton, G. A., \& Phelan, M. C., et al. (2003). Molecular characterisation of the 22q13 deletion syndrome supports the role of haploinsufficiency of SHANK3/PROSAP2 in the major neurological symptoms. Journal of Medical Genetics, 40(8), 575-84. [DOI:10.1136/jmg.40.8.575] [PMID] [PMCID]

Zhang, C., Wu, Z., Hong, W., Peng, D., \& Fang, Y. (2016). Evaluating the association between the SHANK3 gene and bipolar disorder. Psychiatry Research, 244, 284-8. [DOI:10.1016/j.psychres.2016.07.058] [PMID] 